\title{
Las inteligencias múltiples en la clase de educación física; una experiencia formativa con alumnos de educación primaria Multiple intelligences in physical education class; a formative experience in primary school students \\ Guadalupe del Rosario Martínez-Aguilera \\ Secretaría de Educación del Gobierno del Estado de San Luis Potosí, Universidad Iberoamericana León (México)
}

Resumen. Este trabajo buscó descubrir de qué manera la clase de educación física puede favorecer la manifestación de las inteligencias múltiples en la resolución de problemas en 27 alumnos de ocho a nueve años de un grupo de cuarto grado de una escuela primaria. Mediante el método cualitativo en la aplicación de una secuencia didáctica de siete sesiones se recogieron datos mediante una evaluación formativa utilizando entrevistas, observación y el cuaderno de educación física que los niños utilizaron a lo largo de la intervención donde expresaron parte de su experiencia y aprendizajes que adquirieron en la clase de educación física. Como resultado se observa que los alumnos expresan de manera específica los aprendizajes obtenidos en la secuencia didáctica en donde se manifiestan las inteligencias múltiples para la resolución de problemas permitiendo conseguir los objetivos de la secuencia didáctica. Se concluye la importancia de fortalecer una clase de educación física inclusiva mediante el enfoque de las inteligencias múltiples en donde los alumnos puedan expresar sus habilidades y capacidades para lograr los aprendizajes esperados de educación física, se enfatiza la necesidad de trabajar las competencias didácticas en los docentes para planificar escenarios aptos y estimulantes que permitan en el alumnado lograr un desarrollo integral.

Palabras clave: Educación física; inteligencias múltiples (IM); estrategia didáctica; evaluación formativa; escuela primaria.

\begin{abstract}
This work sought to discover how physical education class can favor the manifestation of multiple intelligences in problem solving in eight- to nine-year-old elementary school students. Through the qualitative method in the application of a didactic sequence of seven sessions, data was collected through a formative evaluation using interviews, observation and the physical education notebook that the children used throughout the intervention where they expressed part of their experience and learning that acquired in physical education class. As a result, it is observed that the students express in a specific way the learning obtained in the didactic sequence where multiple intelligences are manifested for solving problems, allowing to achieve the objectives of the didactic sequence. The importance of strengthening an inclusive physical education class through the approach of multiple intelligences is concluded where students can express their abilities and capacities to achieve the expected learning of physical education, the need to work on didactic skills in teachers is emphasized to plan suitable and stimulating scenarios that allow students to achieve development.
\end{abstract}

Keywords: Physical education; multiple intelligences (MI); didactic strategy; formative assessment; primary school.

\section{Introducción}

¿Qué es lo que consideramos inteligente? En décadas pasadas, en el ámbito educativo ser competente en habilidades lógico-matemáticas y en comprensión lectora vislumbraba para educadores y no educadores que un alumno tenía capacidades cognitivas grandes en comparación de los que no tenían altas notas (Gardner, 1995). En el contexto escolar hay determinadas tendencias en el tratamiento del curriculum respecto a la intervención pedagógica que hace el docente y que no permite la manifestación de las inteligencias diversas de los alumnos por los planteamientos didácticos que se le ofrecen

Fecha recepción: 10-05-21. Fecha de aceptación: 30-12-21

Guadalupe del Rosario Martínez Aguilera

maguilera_gpe@hotmail.com por ejemplo el modelo - tradicional-sumativo- que refieren Barrientos, López-Pastor y Pérez-Brunicardi (2019), por ello la necesidad de crear en las escuelas ambientes diversos de enseñanza-aprendizaje que den insumos al alumnado para crear una cultura en el que se valoren las potencialidades individuales de los niños y adolescentes en su etapa formativa.

Tal como lo refiere Garduño (2020) el profesorado ha intentado cambiar las maneras de intervenir en su práctica pedagógica dejando atrás aquellos estilos de enseñanza que evocaban una instrucción directa en donde el educador era el principal protagonista de la sesión (dar órdenes mientras que el alumnado solo obedecía), no obstante, sigue siendo éste uno de los grandes retos en el diseño de las programaciones de los educadores físicos; proponer en su clase un espacio en donde a través de sus estrategias, actividades sugeridas, materiales y recursos, organización del espacio y alumnado etc., 
permite que los alumnos puedan manifestar sus diversas inteligencias de manera inclusiva, es decir favorecer su pensamiento divergente y la capacidad de solucionar problemas con las habilidades y capacidades que poseen.

Gardner (1995) explica que el concepto de inteligencia es la capacidad para solucionar problemas y crear productos que sean valiosos para su cultura, es decir que, «cada persona está dotada de una inteligencia formada a su vez, por una combinación de inteligencias múltiples que varían en grado y profundidad, pudiendo todas ellas poder ser aumentadas con la práctica» $(\mathrm{Ca}-$ rrillo y López, 2014, p. 81). En ese sentido, hemos de reconocer que todos tenemos distintas habilidades y capacidades que nos ayudan a afrontar los quehaceres de la vida, lo que representa uno de los objetivos o metas educativas del siglo XXI, lograr no solo aprendizajes significativos, sino que también puedan resolver problemas en cualquier contexto. Para ello es importante que en los centros educativos estimulen a los estudiantes a demostrar sus distintas habilidades para que sepan que las tienen (es decir, identifiquen que son buenos en algo), que puedan fortalecer las que no han sido antes estimuladas y que se potencien las que se encuentran más desarrolladas por naturaleza.

Desde el área de la educación física puede crearse este ambiente al que nos referimos, donde el niño puede practicar sus habilidades y capacidades de manera integral, se determina que hacer posible esta meta se encuentra de manera nodal en el ejercicio de planificación que el docente realiza, pues es aquí en donde se determina la metodología de enseñanza, con ello, las estrategias didácticas que ha de trabajar en la sesión y cómo ha de llevar a cabo la evaluación formativa que nos permitirá reflexionar sobre la práctica docente implementada y los aprendizaje adquiridos por los alumnos.

Asqui (2017) en su estudio respecto a la influencia de las inteligencias múltiples en el área de la educación, refiere que «el docente ha de tener las habilidades precisas para desarrollar los contenidos y elaborar estrategias acordes a las inteligencias más presentes en sus alumnos esto es, proporcionando alternativas para aprender una materia» (p. 4), no obstante, hemos de mencionar que poner atención solo a unas inteligencias puede resultar redundante a que prioricemos unos saberes más que otros, por lo que hemos de proporcionar un contexto en donde el alumno pueda participar activamente manifestando a cómo le sea posible sus habilidades y conocimientos en la resolución de problemas sin limitar y favoreciendo las habilidades que no se estimulan en una clase tradicional. Este trabajo de investigación que se presenta a continuación corresponde a la pregunta de investigación: ¿De qué manera la clase de educación física puede favorecer la manifestación de las inteligencias múltiples de los escolares en la resolución de problemas? Por lo que el propósito de este artículo es identificar los aprendizajes manifestados por los alumnos en la implementación de una propuesta didáctica que permite valorar los alcances de la clase de educación física bajo el enfoque y los principios de la teoría de las inteligencias múltiples.

\section{Marco teórico}

El enfoque educativo de esta teoría nace a través del investigador Gardner que después de una larga investigación por los años 80's concluye que podemos demostrar nuestra inteligencia de distintas formas, explica que las personas estamos dotadas por ocho diferentes tipos de inteligencias, y que en nuestra capacidad para resolver los problemas que se nos presentan hacemos uso de una combinación de todas o algunas de ellas puesto que todos tenemos diferentes intereses y capacidades. A modo, de síntesis éstas son: lingüística, que hace referencia a la capacidad de manejar el lenguaje oral y escrito; lógica-matemática, se manifiesta el realizar hipótesis y comprobar resultados, donde realza la capacidad de razonar para resolver las situaciones sobre todo del tipo lógico; espacial, donde domina la interpretación de información a través de imágenes y medios gráficos; corporal-cinéstesica, que son el conjunto de habilidades motrices para expresar o ejecutar movimientos complejos puesto que el cuerpo es un eje importante en la manifestación de mensajes; musical, se vincula al dominio del ritmo y la interpretación musical; naturalista, es la capacidad de identificar y apropiarse de elementos de la naturaleza y el medio ambiente; interpersonal, facilita relacionarse con los demás y las personas se distinguen por sobresalir en aspectos sociales e; intrapersonal que refiere a la capacidad para comprender los sentimientos propios como el de otros, manejar las emociones y reflexionar ante la toma de decisiones (Gardner, 1995).

Es importante mencionar que Gardner (1995) ha explicado que las inteligencias múltiples son una manera de entender cómo el ser humano puede resolver problemas de diversas maneras haciendo una combinación de diversos conocimientos y habilidades que innatamente o por estimulo ha desarrollado y fortalecido a lo largo de su experiencia humana pero que no 
podemos determinar, caracterizar o etiquetar a las personas con una inteligencia u otra, ejemplo es, que a manera de que la persona se familiarice con estas inteligencias el propone en su libro Inteligencias múltiples. La teoría en la práctica (1995) un cuestionario en el que podemos acercarnos a conocer que tipo de afinidad tenemos a ciertas inteligencias dado nuestros intereses y gustos, no obstante, esto no puede ser considerado como un diagnóstico para catalogar qué tipo de inteligencia tenemos, puesto que todos tenemos todas las inteligencias en mayor o menor medida.

Hoy en día la educación física centra su objeto de estudio a través del movimiento trabajando con la filosofía de ver a la persona como unidad y globalidad, desde algunos Planes de Estudio en México como el del 2009, 2011 y el actual 2017 precisan en posicionar al alumno como el protagonista de la sesión que explora sus posibilidades, fomentando su creatividad a través de la herramienta principal didáctica del juego. Para la Secretaría de Educación Pública (SEP) la educación física «es una forma de intervención pedagógica que contribuye a la formación integral de niñas, niños y adolescentes al desarrollar su motricidad e integrar su corporeidad» (SEP, 2017, p. 161), cabe recalcar que existe una diversidad de metodologías que se pueden implementar con el fin de que a través del trabajo docente los alumnos desarrollen sus competencias motrices y sean capaces de resolver problemas de distintas maneras.

La didáctica en este sentido, resulta fundamental para determinar la gestión de la clase en cuanto al conocimiento práctico que conjuga la teoría y la acción hallados en los procesos de experimentación e innovación del currículum (Zagalaz, 2003). Pero ¿a qué nos referimos como didáctica? En particular para González (2009) las estrategias didácticas son declaraciones de intenciones respecto a la metodología para llevar a cabo el proceso de enseñanza-aprendizaje, por otra parte, se considera que son «herramientas pedagógicas que permiten un aprendizaje más profundo y permanente, además de propiciar el desarrollo del pensamiento crítico, de habilidades cognitivas y actitudes desde una perspectiva constructivista y significativa» (Morales \& Pereida, 2017, p. 69). Dados estos planteamientos podemos considerar que tomar decisiones respecto a las maneras en las que propiciamos aprendizajes se traduce en que el docente deberá desempeñarse acorde a un conjunto de conocimientos, habilidades y actitudes que conforman competencias didácticas; es decir; saber qué son y cuáles son las estrategias que puede implementar, de qué manera y cómo las ejecuta y cómo asume ciertas actitudes y valores en ese desempeño que garantizan una eficaz relación alumno-docente que permite el intercambio proactivo de mensajes y retroalimentación para garantizar que proceso de enseñanza-aprendizaje cumpla su propósito pedagógico.

Siguiendo a Morales y Pereida (2017) explican finalmente que dentro de la estrategia puede haber diferentes actividades indispensables para la consecución de los propósitos educativos, por lo que, «la educación física debe proponer actividades globales, de resolución de casos, de situaciones problema, etc, que generen la necesidad de tomar decisiones...» (González, 2009, p. 106). Coincidimos finalmente, que en la clase de educación física los alumnos «a lo largo de su formación hayan hecho frente a situaciones y problemas abiertos, que les obligue a tomar decisiones, como es previsible que suceda frente a menudo en su vida como ciudadano» (Blázquez, 2017, p. 48), esto sin duda, garantiza que el alumno haya entrenado en un ambiente en el que se presentan situaciones simuladas de la vida cotidiana en donde no hay maneras específicas, rutas o planes determinados para resolver un problema dado que estos se presentan en contextos diversos, complejos y únicos.

Respecto a la teoría de Howard Gardner, podemos decir que la aplicación del enfoque de las inteligencias múltiples en el ámbito educativo es cada vez más utilizado, ejemplo es, que se ha podido aplicar como referencia desde la organización de los contenidos de enseñanza a través de la taxonomía de Bloom, tal como lo refiere Armstrong (2009), en donde a través de la organización de los objetivos educativos en una planificación se pueden ir favoreciendo el uso de las diversas inteligencias pasando por cada uno de los niveles que corresponde a la taxonomía. Por otro lado, existen algunos trabajos que rescatan el tema de aplicar las aportaciones de Howard Gardner al área de la educación física (Asqui, 2017; Bermúdez, 2019; Cruz y Cruzata, 2017), sin embargo, hacen prácticas o intervenciones que afirman que determinadas prácticas ayudan a desarrollar o trabajar específicamente unas inteligencias lo que no ayuda a estimular el resto de ellas, por ejemplo, el hecho de practicar solamente futbol y no otras actividades, es decir, está claro que por ende la inteligencia más favorecida será la kinestésica. Por ello, en ese sentido, Ubago et.al. (2018) refiere que en realidad «se han hecho pocas investigaciones que tengan como eje temático el estudio de las inteligencias múltiples en relación con la práctica de la actividad física y específicamente en el ámbito educativo» (Ubago, et.al., 
2018, p. 154), lo que abre la posibilidad y la necesidad de seguir profundizando en este tema y rescatar las pocas investigaciones y experiencias sistematizadas en donde se ha utilizado este enfoque en la clase de educación física para difundirlas y reflexionar sus aportaciones.

\section{Planteamiento didáctico y experiencia}

Esta investigación tiene un enfoque cualitativo de tipo descriptivo. De acuerdo a las características cualitativas que aporta Hernández (2014) ésta se basa en la teoría y experiencias iniciales, se orientan hacia la exploración, descripción y el entendimiento de temáticas y por supuesto la relevancia de que los resultados se manifiestan a partir del conocimiento empírico. Respecto a esta investigación, la información deriva de una intervención de la propia docente que constó de una secuencia didáctica conformada por siete sesiones de educación física con alumnos de cuarto grado en una escuela primaria de la ciudad de San Luis Potosí en el 2018.

La muestra constó de 27 alumnos de cuarto grado de primaria, los cuales correspondían a 13 niños y 14 niñas con una edad de 9 y 10 años.

Las técnicas y herramientas para la recolección de los datos fueron entrevistas semi-estructuradas a los alumnos para conocer las nociones que tenían respecto a la clase de educación física y los aprendizajes adquiridos a lo largo de la intervención, la observación participante, el uso del diario del profesor en donde la docente anotó aspectos que consideró relevantes durante las sesiones de educación física con los alumnos, sus expectativas y recomendaciones para la implementación de las actividades subsecuentes y el análisis documental de los cuadernos de los niños para identificar los aprendizajes que los alumnos adquirían; los sentimientos y opiniones derivadas de su participación en la clase de educación física a través de preguntas abiertas, listas de cotejo y dibujos que los alumnos plasmaron a lo largo de la intervención. El proceso de recolección y análisis de los datos le llevó a la docente tres meses y una vez terminado dicho proceso organizó los resultados en un escrito de corte narrativo.

\section{Organización didáctica de la secuencia}

El objetivo de la secuencia didáctica fue que los alumnos reconocieran la importancia de diseñar estrategias de juego manejando sus capacidades físico motrices aso- ciando su pensamiento y acción para la solución de problemas. Esta intervención constó de siete sesiones de cincuenta minutos cada una dentro de una secuencia didáctica que se describe a continuación:

1. Sesión 1 «Qué es una estrategia?»: El propósito de esta sesión fue que el alumno conociera y comprendiera el concepto de estrategia por medio de actividades lúdicas. Como estrategia didáctica se utilizó el rally con distintas tareas que exigía el uso de distintas habilidades para conocer el concepto de estrategia. Los alumnos trabajaron en pareja resolviendo las siguientes tareas; armar un rompecabezas, dar una marometa en un colchón, saltar un bebeleche, cuestionar a otros compañeros del grupo sobre el tema de la sesión, buscar un objeto de la naturaleza por el espacio escolar, resolver un gato e identificar un logro. Al lograr cada reto los alumnos ganaban un papel que contenía una palabra misma que al juntar todas conformaba un enunciado sobre el término de estrategia, al finalizar la sesión los alumnos escribirían junto con su pareja una definición de estrategia con apoyo de las palabras recolectadas.

2. Sesión 2 «El juego y la estrategia»: El propósito fue que el alumno asociara la estrategia como un elemento fundamental en la resolución de problemas de juego. Se utilizó la estrategia didáctica del juego motor. «Escape imposible» fue un juego que consistió en que los alumnos se ubicaran en distintas posturas de manera entrelazada unos con otros en un espacio reducido para no moverse en un tiempo que era definido por la profesora, terminando el tiempo cada uno ideaba la forma de salir sin tocar a los compañeros. «El cielo de globos» fue un juego que consistía en crear estrategias para que los globos que ellos mismos golpeaban hacia arriba no cayeran al suelo, pues de alguna manera todos los globos debían estar en el aire mientras que el profesor daba distintas consignas que aseguraba que ningún alumno se quedara en un mismo lugar, un ejemplo de consigna era que, mientras el globo estaba en el aire un alumno tenía que ir a saludar a un compañero antes de que cayera de distinta manera y con un compañero distinto cada vez.

3. Sesión 3 «Pienso y me muevo»: El propósito fue que el alumno maneje sus capacidades físico-motrices en beneficio de la actuación estratégica durante los juegos. Mediante la estrategia didáctica del juego modificado se realizó la actividad titulada «Pelota baja» misma que corresponde a la clasificación de cancha dividida. El grupo fue dividido en dos equipos, mientras que el espacio de cada uno es la mitad de la cancha escolar, ambos se enfrentan lanzando pelotas de plástico al espacio del equipo rival mientras que al mismo tiempo no de- 
ben dejar pasar pelotas en la línea final de la cancha (es decir, de su espacio), pues mientras más haya pelotas en su espacio al final de un tiempo límite es prueba de que el equipo ha perdido una partida.

4. Sesión 4 «Pensemos antes de actuar»: El propósito fue que el alumno solucionara problemas de juego asociando sus conocimientos para después inferir en las tareas. Las actividades de «Asaltando Pañuelos» y «La casita» que figuran estrategias cooperativas constó en que alumnos trabajaron todos juntos por grupos para conseguir el mayor número de pañuelos posibles en el tiempo límite sin que les quitaran los suyos, por otra parte, con tapetes didácticos de foami los niños formaron casitas las cuales fueron andamios para hacer reflexionar al alumno el análisis de las decisiones tomadas al realizar una construcción imaginativa de una casa con los materiales.

5. Sesión 5 «Construimos la estrategia»: Tuvo como propósito que el alumno propusiera formas intencionadas para favorecer la resolución de problemas. Se utilizaron estrategias didácticas de dinámica grupal y juegos de ritmo musical. «Bobby» (un peluche) fue la presentación simbólica de una situación de conflicto con una persona a quién tenían que idear maneras de saludar y lograr ser su amigo mientras el peluche -Bobby- estaba indispuesto y molesto, cada alumno presentaba maneras de intentar socializar con él y lograr ser amigos. El juego de las «Las olas de mar» por otra parte, implicaba mantener el ritmo corporal de acuerdo a la música mientras manipulaban una sábana e intentando a su vez que la pelota sobre ella no se cayera al suelo; el ritmo musical fue variando presentando así niveles de dificultad distintos y poniendo consignas respecto al movimiento del equipo por el espacio en el que nos encontrábamos.

6. Sesión 6 «Puedo solucionar problemas»: Se propuso que el alumno demostrara un dominio en explicar cómo ha resuelto el problema de las actividades propuestas. Los alumnos dominaron la elaboración de planes o construcciones de modelos para solucionar los problemas en la actividad cooperativa «El gran Jenga». Fue llevado el popular juego de mesa a gran escala con cajas de cartón, en dónde se saca de una torre fichas (cajas) que se van colocando arriba resultando así cada vez más difícil de sacar cajas sin que ésta se derribe. Se conformaron tres equipos en donde de manera colaborativa los alumnos jugaron jenga y establecían diversas maneras de jugarlo, acordando reglas y acuerdos entre ellos.

7. Sesión 7 «Reconociendo mis habilidades»: El propósito en esta sesión fue que el alumno practicara las diversas capacidades y habilidades aceptando y recono- ciendo la de los otros en la resolución de problemas. Se utilizó la estrategia didáctica del circuito de acción motriz (al que se le tituló «Exatlón»). Tras la participación de diversos retos motores dentro del circuito los alumnos se autoevaluaron y se hizo una reflexión sobre los aprendizajes que mostraron al inicio de la unidad en la implementación del rally contrastando con los aprendizajes que hasta este momento final lograron adquirir. Algunos de estos retos eran que por parejas (amarrados de su pierna derecha y pierna izquierda respectivamente), tenían que pasar conos en zig-zag, pasar por arriba de una vaya, lanzar un aro a un objetivo definido, reptar mediante un túnel y finalizar pateando un balón a una potería.

Para visualizar las actividades implementadas y las planeaciones detalladas y completas de esta secuencia se encuentran en el apartado de anexos en el trabajo de Martínez (2018).

\section{Resultados de la experiencia; la educación} física como medio para fortalecer la resolución de problemas a través de las inteligencias múltiples

\section{Evaluación inicial}

Como primer momento durante la evaluación inicial respecto al tema que se estaría desarrollando y al hacerle algunas entrevistas a los niños para saber sus concepciones y experiencias previas a la sesión de educación física, una de las preguntas más significativas para analizar los resultados de esta investigación fue preguntarles «¿Qué es la educación física para mí?» Las respuestas se categorizaron de acuerdo a lo que escribieron en las libretas, al no ser preguntas cerradas los porcentajes de cada una se basaron en relación con lo que expresó la mayoría del grupo; el 58.3\% de los alumnos veían la clase de educación física como un espacio de diversión o entretenimiento; el 37.5\% mencionó que solo era un tiempo para salir a jugar; el 20.8\% señaló que era un espacio para convivir y jugar con los amigos, en este mismo porcentaje también dijeron que se veía deporte, como el practicar balonmano o futbol; mientras que el $16.6 \%$ refirió que en aspectos de salud servía para mejorarla y estar sanos y; en una minoría con el $4.1 \%$ dijo que era tiempo libre y oportunidad para aprender a reciclar, cuidando el medio ambiente. Lo importante de esta pregunta es que solo el $12.5 \%$ escribieron en sus libretas que nos ayudaba a aprender algo.

Otra pregunta relevante fue ¿Qué es lo que he apren- 
dido en la clase de educación física a lo largo de los años? Con el 29.1\% mencionó que cuidar el medio ambiente y los valores (el trabajar en equipo, aprender a respetar, entre otros), otro porcentaje elevado con el 20.8\% dijo que ha aprendido a jugar, mientras que el 12.5\% mencionaron que a ejercitarse y divertirse; el 8.3\% ejemplificó que han hecho manualidades y en una minoría con el $4.1 \%$ comentaron que saber ganar y perder, coordinar y aprender algún deporte.

Un aspecto a destacar de las preguntas que se hicieron es que al pedirles su concepto de inteligencia o lo que opinan al respecto fue que el $41.6 \%$ lo asociaron con aprender; el 25\% dijo que las personas inteligentes eran las que saben cosas; el 12.5\% mencionó que son los estudiosos, que era cuando algo lo hacías bien y ser listos; el $8.3 \%$ escribieron que eran las personas que eran muy sabias o tener sabiduría; y con el $4.1 \%$, expresaron que eran los que son buenos en una materia, los que lograban algo o que hacían las cosas rápido como una computadora.

Posterior a conocer sobre sus concepciones sobre el tema y la educación física interpretando que la mayoría de sus respuestas se relacionaban al hecho de observar que los que sacan mejores calificaciones se asume que son los más inteligentes; descubrimos cómo ligaban dichas habilidades y capacidades a la práctica de la educación física por medio de un dibujo. Se les explicó que debíamos de dibujar el contorno de nuestra mano en la hoja y que en cada uno de los dedos escribieran algunas habilidades que ellos creían que en la clase usaban, fortalecían o aprendían, conforme pensaban a algunos se les dificultó reconocerlas, ellos expresaban: «Maestra, es que no sé qué poner», «Maestra, me faltan dedos por llenar ¿Qué hago?»o «No sé cómo hacerlo».

Gran parte del grupo no pudo reconocer las habilidades que la clase de educación física les ayudaba a fortalecer; en gran parte de las manos dibujadas tuvieron que ser rellenadas por gustos que no correspondían a lo que se hacía en la escuela, esto evidenciado con que algunos pusieron cosas como «me gusta la pizza»o «me gustan los videojuegos» mientras que otros optaron por repetir cualidades dos veces por rellenar la mano y no quedara incompleta además hubo otros pocos que dejaron dedos vacíos al no saber que escribir. Aquellos que si lo relacionaron con algunas habilidades fueron frases como «correr» «bailar» «futbol» «basquetbol» o generalizaron con «me gusta jugar» rellenando con frases de «me gusta hacer amigos» «me gusta estar con mi familia» entre otros.

\section{Evaluación intermedia}

Durante el desarrollo de la secuencia didáctica analizando su cuaderno encontramos que hubo aprendizajes en relación a que los alumnos reconocían como en la clase educación física es un espacio en el que se aprenden distintas habilidades y no solamente aquellas que se relacionan específicamente a la salud física, reflexionando sobre lo que habían aprendido y las habilidades que tuvieron que usar y fortalecer para resolver los retos propuestos. Algunos testimonios que se dieron a lo largo de la intervención relacionadas a las concepciones de la asignatura sobre lo que aprendieron en ella fueron las siguientes:

Alumno J: Algo que he aprendido en la clase de educación física es hacer manualidades. La educación física me ayuda a pensar cómo hacer cosas y poder conseguirlo».

- Alumna K: La educación física es un tiempo libre de convivir con nuestros compañeros y con la maestra. He aprendido a reciclar, a cuidar el medio ambiente, a seguir patrones y a coordinarnos»

Alumna A: He aprendido a no pelear. La inteligencia es algo que nos hace pensar y la clase me ayuda porque a prendemos a convivir y no pelear con los otros, también aprendemos a reciclar materiales.

- Alumno P: La educación física para mí es convivir con mis compañeros, he aprendido a trabajar en equipo y me ayuda a ser inteligente porque me ayuda a reflexionar todo

Durante la implementación del rally en la primera sesión se encontró que los alumnos asociaron la clase de educación física como un espacio que les estimula la capacidad de reflexionar y pensar situaciones, ejemplo fue que en los cuadernos los alumnos expresaron:

Alumna K: Aprendí a busca pistas, todas se me hicieron fáciles, más la de los colchones porque yo soy muy ágil con la gimnasia

Alumna E: Aprendí a seguir reglas como la de la hoja, trabajamos muy bien, me sentí bien con las actividades que unas eran difíciles y otras fáciles.

- Alumna M: Aprendimos a descifrar cosas pensando y jugando bien divertido

En la sesión donde se intentó que los alumnos empezarán a asociar la estrategia como elemento para darle solución a los problemas motrices con estrategias didácticas del juego motor como «Cielo de globos» $\mathrm{y}$ «Escape Imposible», los planteamientos aludidos fueron:

Alumno J: Mi estrategia fue voltear para no tocar al otro y salir lentamente, además de que tuve que tener precisión.

- Alumna K: Aprendí a ponerme de acuerdo con alguien más y hacer las cosas bien y correctas y cómo calcular los 
obstáculos que nos pongan. Tuve que resbalarme lentamente y fijarme alrededor de no tocar a mis compañeros.

Alumna E: Aprendí que la estrategia es que si no puedes hacer algo tienes que inventarte algo más por ejemplo maneras diferentes de salir sin que los compañeros me tocaran.

Alumna A: La estrategia me permite hacer movimientos y diferentes posiciones, algo que utilicé en el juego. Tuve que hacer ideas y estrategias para moverme y que no se cayera el globo.

Alumno P: Aprendí a que debo estar atento porque tuve que mirar el globo para que no se cayera.

En la aplicación de estrategia didáctica relacionadas el juego modificado, de expresión corporal y dinámicas de socialización los alumnos expresaron:

- Alumna A: La clase me ayuda a mejorar mis habilidades porque hacemos varias cosas que antes no podíamos hacer, como correr y compartir en parejas. Aprendí con el juego a querer las personas, a preguntarle a los demás qué les pasa y cómo puedo arreglarlo.

Alumno P: Pusimos estrategias porque planeamos las posiciones que teníamos que hacer como equipo para poder movernos.

- Alumna M: Me ayudó la clase a entender qué es una estrategia porque me dan pistas para poder comprender, me gusta la clase porque me relaja al moverme.

- Alumno J: Aprendí a resolver problemas cuando se es honesto y si alguien discute, tengo que decirle ¿qué paso? y ¿Quién empezó?

- Alumna K: Aprendí lo que es ser compartido al jugar. Si hubiera una discusión yo primero les diría que dialoguen y que tienen cosas en común, tranquilizarlos, calmarlos para que no haya violencia.

Alumna C: Para resolver el problema, le hice caso a una compañera que dijo que cuando fuera la pelota a un lado la aventáramos a la esquina para que no se cayera, yo dije que pusiéramos la sábana recta y funcionó perfectamente.

\section{Evaluación final}

Finalmente, en las últimas sesiones, cuando se aplicaron las actividades de «El gran Jenga» $\mathrm{y}$ «El Exatlón» los estudiantes hicieron planes donde identificaron elementos que debieron de tomar en cuenta para haber solucionado el reto que se les presentó. Se manifestaron en los escritos las habilidades y características de las diferentes inteligencias que los niños tienen y cómo de una manera personal las combinan para haber resuelto el problema, por ejemplo, algunas oraciones que escribieron en sus cuadernos fueron:

Alumna K: Lo que hice fue deslizar lentamente para que se saliera, moverme lentamente; veía cual pieza podía sacar; unos lo hacían muy bien, pero otros les faltó precisión, yo calculaba para que no se cayeran las demás.

Alumna E: Yo en el juego tomé en cuenta para jugar que debía estar tranquila, tener paciencia, generar una estrategia, pensar y ser cuidadosa.

Alumna R: Para resolverlo, me tuve que fijar si se movía o no el jenga, mover algunas piezas del jenga para saber cuál se movía más y cuál menos, también no tenía que apresurarme, no distraerme cuando era mi turno y, por último, no apresurar a mis compañeros.

Alumna A: Tuve que considerar mi vista, mi tacto, equilibrio y ver cual estaba más floja para sacarla.

Alumno P: Para jugar tuve que golpear los jengas para ver si alguna estaba floja, después tuve cuidado que no se cayera y le tenía que decir a los otros compañeros cual podía sacar.

Alumno J: En el juego tuvimos que cooperar, por ejemplo, es ayudar a los que no pueden o como en la calle un señor tiene una taza y le doy moneditas, la cooperación es ayudar a cuidar perritos, gatitos y personas callejeras.

- Alumna C: Trabajamos en equipo para resolver y ayudamos a otros, aprendimos a confiar, a tener seguridad y de habilidades agilidad, correr rápido para resolverlo lo más pronto posible.

Alumna M: Aprendí en el juego de Jenga que la cooperación es que todos ayuden a el equipo para sacar mejores resultados. En el juego de exatlón lo que practicamos fue el compañerismo, la amistad y el trabajo, por lo que cooperamos apoyando al equipo para poder ganar y ser mejores.

Como lo refiere Martínez (2018), la autoevaluación desde el enfoque formativo busca que los alumnos sepan y aprecien sus procesos de aprendizaje y sus acciones para que cuenten con elementos para mejorar, en este sentido, contrastar sus conocimientos y trabajos de evidencia iniciales con los resultados finales reconocieron qué y cómo fue lo que aprendieron en la clase de educación física al exponer los pasos para solucionar los problemas y cómo fueron asociados con el uso de las diversas habilidades y capacidades que finalmente constatan el supuesto de que todos tenemos las inteligencias múltiples en mayor o menor medida y que la oportunidad de poder expresarlas para fortalecerlas con la práctica dependerá del estímulo que el ambiente de la clase proporciona así como ocurrió en esta intervención.

\section{Reflexiones sobre la experiencia en la in- vestigación}

Actualmente el plan de estudios para la escuela primaria en México declara la importancia del desarrollo 
integral para formar a los ciudadanos que la nación requiere y necesita, no obstante, muchas veces existen brechas entre estos planteamientos y las maneras en las que se planifica la acción pedagógica que el docente sigue y más aún el cómo se traduce esas secuencias didácticas a la situación real con los niños en las aulas y canchas escolares. El enfoque de las inteligencias múltiples da la posibilidad de repensar y reconstruir la manera en la que se enseña y como el niño aprende, pues a pesar de que existen contenidos específicos que los profesores quieren lograr durante el proceso de enseñanza-aprendizaje es posible favorecer que se expresen habilidades sociales, pensamiento lógico-matemático, de regulación emocional, expresión artística, autoestima e incluso empatía al cuidado del medio ambiente tal cómo se vio reflejado en esta investigación, que al tomar el juego como estrategia básica principal permitió que el niño pudiera expresar distintas habilidades combinadas entre sí para resolver los problemas pues «los juegos son mecanismos básicos del proceso de socialización fundamentados en las características de la cultura en la que se inscriben» (Casadiego, 2021, p. 241); lo que refiere a que en la manera en la que nosotros orientemos el juego y se potencializa para que sea un espacio libre de expresión será significativo para el estudiante.

En esta investigación didáctica al trabajar bajo el enfoque de las inteligencias múltiples propició que los alumnos reforzaran la capacidad de resolver problemas al pensar maneras distintas para resolver los retos presentados en las clases, mismos que se vieron reflejados en los testimonios de los niños en sus libretas, ya que «el cuaderno es una representación de cómo el alumno interioriza esos conocimientos, los contenidos que se enseñan y cuál es la metodología seguida por el maestro» (Fernández, 2017, p. 78.), lo que claramente es una manera de afirmar que la observación mediante una evaluación formativa es la manera ideal para poder ver cómo los alumnos van construyendo su aprendizaje, es decir, los exámenes no son las maneras de evaluar lo que el alumno ha aprendido pues esto lo convierte en un instrumento tajante que no propicia ni fomenta la expresión libre del educando.

La clase de educación física en ese sentido se puede resistir a la idea de utilizar un cuaderno de clase, no obstante, es una de estas alternativas de evaluación formativa en las que permite recoger los datos que los niños muchas veces en las clases no pueden expresar en sus comentarios ante el tiempo limitado que tiene el profesor con los niños en las escuelas, es por eso que «puede verse como un lugar donde el estudiante puede, por ejemplo, recoger los diferentes intentos o etapas seguidas al desarrollar una actividad o problema, comunicar sus ideas y reflexionar sobre ellas, reflexionar sobre su propio trabajo, aprendizaje y comprensión» (Arce, 2018, p. 371), lo que en este trabajo de investigación se realizó y que permitió recoger la experiencia de los niños en toda la intervención, es decir, que este recurso fortalece el planteamiento de que bajo el enfoque de las inteligencias múltiples, las secuencias didácticas se deben de plantear en razón de potenciar el aprender a pensar tal como lo refiere García (2012). Añadimos que, no basta con plantear un mismo reto desde distintas posibilidades sino también ver la manera en la que los niños pueden expresar el proceso que siguieron al resolverlo lo que podría ayudar a reconocer en sí mismos sus capacidades de resolver problemas bajo el uso de sus inteligencias múltiples, tal cómo se reflejó en este estudio en donde evidentemente reflejaban en sus escritos el cómo utilizaban sus distintas habilidades y conocimientos en la clase de educación física.

\section{Conclusiones}

Este trabajo expone la necesidad de darle importancia al fortalecimiento de las competencias didácticas del profesorado para diseñar y organizar distintas actividades de manera creativa y coherentes a la metodología y objetivo educativo, que sea un espacio atractivo para los alumnos y que genere procesos metacognitivos al representar desafíos intelectuales interesantes. Concordamos con Díaz Barriga (1999) al referir que una oportunidad que tienen los maestros es que puedan expresar saberes y las capacidades intelectuales que poseen para mejorar el desempeño en el área que desempeñan, pues «el programa opera en la realidad escolar sólo a partir de la actividad intelectual del docente» (p. 66), es decir, el docente deberá propiciar insumos y andamios para que analicen, reflexionen y creen estrategias durante las sesiones puesto que distintas actividades representan distintas maneras de resolver problemas.

Trabajar bajo el enfoque de las inteligencias múltiple permite que de manera abierta y flexible los niños expresen habilidades y capacidades que a veces no son estimuladas en los ambientes de clase y fuera del entorno escolar cuando el contexto o los padres de familia no estimulan otros saberes y habilidades, más aún, no los valoran. Este trabajo aporta a la comunidad científica una orientación para poder llevar a los alumnos más oportunidades para desarrollar sus habilidades intelectuales de manera lúdica; cualquier propósito educativo 
y contenido del curriculum escolar puede ser trabajado favoreciendo la formación integral del estudiante, donde se concluye con evidencia que el juego se convierte en un fuerte aliado en el desarrollo del pensamiento.

Es importante erradicar el supuesto que lo más importante de una sesión es la parte medular; toda una sesión desde su parte inicial a la final debe valorarse pues son piezas clave para formar el rompecabezas global de los procesos de enseñanza-aprendizaje. Concordamos con Chiva, Capella y Pallarés (2018) en que «la docencia y la investigación educativa no son actividades distintas» (p. 291). Es necesario reconocer la importancia del trabajo reflexivo por parte del profesional en la educación para mejorar la práctica y fortalecer las competencias didácticas que ayudarán en consecuencia a favorecer la formación integral del estudiante cuando se diseñan ambientes aptos y estimulantes a todas las inteligencias.

\section{Referencias}

Armstrong,T. (2009). Inteligencias Múltiples en el aula.Guía práctica para educadores. México D.F.:Paidós Mexicana.

ArceS., Matías (2018). El cuaderno de matemáticas: uninstrumentorelevante en las aulasque suele pasar desapercibido. En La Gaceta de la RSME, 21 (2), 367-387. https:// documat.unirioja.es/servlet/articulo?codigo $=6488240$

Asqui Luna,J.et.al.(2017). Influencia de la teoría de lasinteligenciasmúltiples enla educación física: estudiode casos. En Revista Cubana de Investigaciones Biomédicas, 36 (3), 1-12. http:// scielo.sld.cu/pdf/ibi/v36n3/ibi12317.pdf

BarrientosH., E., LópezPastor,V., \& Pérez-Brunicardi,D. (2018). ¿Por quéhago evaluaciónformativa y compartiday/o evaluación para el aprendizaje en EF? La influencia de laformación inicialypermanente del profesorado. Retos, 36, 37-43.https:/ /doi.org/10.47197/retos.v36i36.66478

BermúdezT., Carlos \& Sáenz-López, Pedro (2019) Emocionesen Educación Física. Unarevisión bibliográfica (2015-2017). En Retos, 36(1), 597-603.

BlázquezS., D (2017). Cómoevaluar bien. Educación Física. El enfoque dela evaluación formativa. Barcelona: INDE.

Carrillo G.,María E.y LópezL.,Amando(2014). Lateoría de las inteligencias múltiples enla enseñanza de las Lenguas. En Contextos Educativos, 17(1), 79-89. https:/ / dialnet.unirioja.es/ descarga/articulo/4690236.pdf

Casadiego, et. al. (2021). Logros de niños y niñas de educación inicial mediante el juego conbloquesde Lego. En Retos, 40(1), 241-249.https://recyt.fecyt.es/index.php/retos/article/ view/78802/62623

Chiva Bartoll,Ò.; Capella Peris, C.\& Pallarés Piquer, M.(2018). Investigación-acción sobre un programa de aprendizaje-servi- cio en la didáctica de la educación física. Revista de Investigación Educativa, 36(1), 277-293.

Cruz O.,Alda\& Cruzata M.,Alejandro (2017). Inteligencia emocional y kinestésica en la educación física de la educación primaria. En Actualidades Investigativas en Educación, 17 (2), 1-20. https: / / www.redalyc.org/articulo.oa:id $=44758530012$

Díaz Barriga, A. (1999). El docente y los programas escolares. Lo institucional y lo didáctico. México, D.F.:UNAM, BonillaArtigas Editores.

Fernández G. Elena (2017). Entre lo impreso y lo manuscrito: viaje por España de la mano de un manual y un cuaderno escolar. En CABÁS- revista digital sobre el PHE, 1 (17), 68-95. http://revista.muesca.es/articulos17/395-entre-lo-impreso-y-lo-manuscrito-viaje-por-espana-de-la-mano-de-unmanual-y-un-cuaderno-escolar

García R., JoséA., (2012). Las secuencias didácticas un área de encuentro entre lasinteligencias múltiples y lascompetencias. En Revista Electrónica Actualidades Investigativas en Educación, 12, (2), 1-30. https://www.redalyc.org/pdf/447/ 44723437016.pdf

Gardner, H. (1995). Inteligencias múltiples. La teoría en la práctica (Traducido al español).México: Ediciones Culturales Paidós.

Garduño D., Jorge; Solís M., Ludibethy Rivera F., Hugo (2020) Estilos de Enseñanza del Concurso Nacional 2017 de la sesión de Educación Física en educación primaria. En Revista Dilemas Contemporáneos: Educación, PolíticayValores, 7 (2), 1-

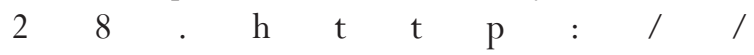
www.dilemascontemporaneoseducacionpoliticayvalores.com/

GonzálezArévalo, C. (2009). Diseño curricular y programación de la educación física en base a las competencias. En Blázquez S.D. (coord.), Enseñar por competencias en Educación Física.Barcelona: INDE.

HernándezS., R. (2014). Metodología de la investigación. Sextaedición. México D.F.:McGraw-Hill Education.

MartínezAguilera, Guadalupe R. (2018). Fortalecer la capacidad de resolver problemas en la clase de educación fisica por medio del uso de lasinteligencias múltiples (Ensayo pedagógico para obtener grado de Licenciatura en Educación Física). BECENE, San Luis Potosí. https://repositorio.beceneslp.edu.mx/jspui/ handle/20.500.12584/65

Morales, R. E. \& Pereida, M. A. (2017). Inclusión de estilos de aprendizaje como estrategia didáctica aplicada en unAVA. En Campushirtuales, 6(1), 67-75.

SEP (2017). Aprendizajes clave para la educación integral. Educación Física.Educación Básica. Plan y programas de estudio, orientaciones didácticas y sugerencias de evaluación. México: SEP.

Ubago J,J.L., et.al. (2018). Relación entrelaTeoría delas Inteligencias Múltiples y laactividad físico-deportiva. Revisión bibliográfica.EnSportis SciJ,4(1), 144-161.

ZagalazSánchez, M. L. (2003). La didáctica de la educación física. En Sánchez Bañuelos, F. (Coord.) Didáctica de la Educación Física para Primaria.Madrid: Pearson Educación. 